Jean-François LAPLÉNIE*

\title{
Lire en allemand, écrire en italien Réception et création à Trieste dans la première moitié du $\mathrm{XX}^{\mathrm{e}}$ siècle
}

This paper aims at showing how aesthetical problems in the literature of a multicultural area are intimately connected with historical and political structures. The lack of an Italian university in Habsburg Trieste forced the young Italians of the city to choose between an education in Arts in Florence and an education in Sciences in Vienna. The tension resulting from this polarisation may explain the lack of "cultural tradition" in Trieste, but also the importance of the choice and the practice of literary language by Triestine writers, a condition of the modernity of this literature.

Hier soll gezeigt werden, wie bestimmte Merkmale der Triestiner Literatur der Jahrhundertwende - wie etwa der Zweifel an der Sprache oder das Interesse an naturwissenschaftlichem Wissen - mit der besonderen politischen Lage der Stadt Triest im Vielvölkerstaat in Zusammenhang stehen. Die sogenannte «italienische Universitätsfrage » hinderte die Triestiner Italiener daran, innerhalb der Habsburger Monarchie eine akademische Ausbildung in italienischer Sprache zu erhalten, und zwang sie, sich entweder für einen geisteswissenschaftlichen Studiengang in Florenz oder für einen naturwissenschaftlichen bzw. technischen in Wien zu entscheiden. Diese Spannung trug zwar zur Identitätskrise der Triestiner bei, schuf aber auch günstige Bedingungen für das Entstehen einer spezifischen Form der ästhetischen Moderne.

Ettore Schmitz, Scipio Slataper, Roberto Bazlen : de nombreux écrivains triestins portent jusque dans leur propre nom l'identité complexe de leur ville. Ettore Schmitz, au prénom grec et au nom juif rhénan, fera même de ce mélange un étendard en prenant le pseudonyme d'Italo Svevo, l' « Italien souabe », nom dont il indique lui-même « qu'il semble vouloir jumeler les races italienne et germanique ${ }^{1}$. Dans son récit $I l$ mio Carso $(1912)^{2}$, Scipio Slataper, au prénom si fortement rattaché à

1. Italo Svevo : Profil autobiographique, in : Écrits intimes, essais et lettres, trad. Mario Fusco, Paris : Gallimard, coll. «Du Monde entier», 1973, p. 13 (traduction modifiée).

2. Scipio Slataper : Il mio Carso, Roma : Editori Riuniti, 1982. Traduction française : Années de jeunesse qui vous ouvrez tremblantes..., trad. nouvelle de Thierry Loisel, Paris : Gallimard, 1996. 
la tradition latine, se met en scène à plusieurs reprises sous le nom de Pennedoro («Plumedor»), traduction italienne de son nom slave. Par leur patronyme et leur parcours, Svevo, Slataper et Bazlen sont représentatifs de ce que Claudio Magris et Angelo Ara ont décrit comme $l^{\prime}$ «identité de frontière ${ }^{3}$ de la ville de Trieste : sa situation géographique, intellectuelle, culturelle, à la frontière des mondes germanique, slave et italien, produit une tension, un vide d'identité. Il y a chez les écrivains triestins, dans leur position sociale comme dans leur écriture, une tension constante entre ce qu'on a et ce qui est loin, entre ce qu'on est et ce qu'on aurait aimé être, entre le lieu réel de la ville portuaire et les lieux mythiques que constituent les idéologies nationales et culturelles.

C'est là presque devenu un lieu commun, un mythe de l'histoire littéraire : une ville et sa difficile quête d'identité - si l'on prête l'oreille à l'étymologie de ce mot tant employé : proprement ce par quoi on est «pareil» (idem). Et de fait, Trieste n'est pas «identique»: ville italienne en territoire slovène et sous domination autrichienne jusqu'au traité de Saint-Germain-en-Laye; ville italienne mais qui souffre de ne jamais l'être complètement, ville qui est et demeure obstinément périphérique par rapport aux centres culturels de la Péninsule. Ce phénomène de décentrement constant se fait sentir de façon particulièrement nette chez les écrivains triestins du début du XX $\mathrm{XX}^{\mathrm{e}}$ siècle. Si, en effet, leur «malheur » aura été, à l'instar de Slataper, « de lire des livres en allemand et d'écrire en italien $»^{4}$, c'est bien que la formation, l'éducation, les pratiques de lecture et d'écriture se lient, dans le cas de Trieste, pour empêcher que les Triestins se sentent «pareils »-idem - et puissent directement participer d'une identité. De fait, Svevo a fait son éducation secondaire en Allemagne, Slataper est un ardent lecteur de Hebbel, d'Ibsen et de Nietzsche, Roberto Bazlen mêle sa prose de nombreux mots allemands qu'il semble considérer comme plus «parlants» que les mots italiens.

Malgré la diversité des « projets culturels » triestins (Claudio Magris), l'hétérogénéité culturelle et linguistique entre ce que les écrivains triestins reçoivent et ce qu'ils produisent semble constituer la marque paradoxale de leur propre identité culturelle. Il s'agit d'analyser ici en quoi cette hétérogénéité, due à la réalité historique et politique du territoire triestin, détermine la réponse profondément originale que les Triestins apportent, au début du $\mathrm{XX}^{\mathrm{e}}$ siècle, aux questions fondamentales soulevées par la modernité. La multiculturalité de Trieste semble déboucher sur son absence de «tradition de culture »; mais il ressort rapidement

3. Angelo Ara et Claudio Magris : Trieste : une identité de frontière, trad. de l'italien par Jean et Marie-Noëlle Pastureau, Paris : Éd. du Seuil, 1991.

4. Cité d'après Michel David: La psicoanalisi nella cultura italiana, Torino: Boringhieri, 1966, p. 379. Je traduis. 
que cette question s'enracine dans le problème particulier de la formation intellectuelle des Triestins, structurée par un double tropisme, vers Florence d'une part et vers Vienne d'autre part. Ville écartelée, ville en recherche et en lutte, ville au contexte spécifique et singulier, Trieste constitue, dans la première moitié $\mathrm{du} \mathrm{XX}^{\mathrm{e}}$ siècle, un laboratoire particulier de la modernité européenne.

\section{L'espace multiculturel triestin et l'absence de tradition culturelle. Autour d'un article de Scipio Slataper}

L'histoire de Trieste est effectivement celle d'une ville-frontière au sens où le politologue Joël Kotek la décrit : une « ville [...] non seulement polarisée sur base ethnique ou idéologique, mais surtout disputée parce que située à la charnière d'ensembles ethniques ou idéologiques », un «lieu d'affrontement» essentiellement politique ${ }^{5}$. Dans le cas de Trieste, ce problème politique remonte à 1382 , lorsque la ville, pour échapper à la menace vénitienne, se "donne » littéralement au duc d'Autriche Léopold III. Devenue autrichienne à la période même où se forment les États héréditaires de la maison d'Autriche ${ }^{6}$, elle le demeurera pendant plus de cinq siècles, jusqu'à ce que le dernier gouverneur autrichien de la ville, Alfred von Fries-Skene, remette le pouvoir, le 30 octobre 1918, entre les mains d'un comité de salut public. Mais ce simple effet de domination politique n'explique pas la complexité de l'espace multiculturel que constitue la ville. En effet, sa situation «au carrefour de plusieurs peuples » ${ }^{7}$ 'a fait d'elle un « creuset » que grâce à l'afflux massif d'immigrants après que l'empereur Charles VI l'eut déclarée port franc en 1719, en même temps que Fiume. Les deux villes deviennent ainsi les principaux débouchés maritimes des territoires sous domination habsbourgeoise, ce qui favorise considérablement leur croissance et leur enrichissement aux dépens de Venise.

L'afflux massif des populations les plus diverses place la ville dans une «situation délicate $»^{8}$, même si la cohabitation entre les diverses communautés se passe sans incident jusqu'en 1848. Toutes - Italiens, Slovènes, Croates, Allemands, Grecs, Juifs - sont unies par le plus puissant des facteurs d'ordre : l'intérêt supérieur du commerce du port. En

5. Joël Kotek : « Les villes-frontières au $\mathrm{XX}^{\mathrm{e}}$ siècle. Être et ne presque plus être », in : J. Kotek (dir.) : L'Europe et ses villes-frontières, Paris : Éd. Complexe, 1996, p. 15-33, cit. p. 17.

6. À titre de comparaison, rappelons que la Carinthie est acquise par les Habsbourg en 1335 et le Tyrol en 1363. Chronologiquement, Trieste est politiquement autrichienne avant le Vorarlberg et bien avant Salzbourg.

7. I. Svevo : Profil autobiographique (note 1), p. 15.

8. Roberto Bazlen : Scritti, a cura di Roberto Calasso. Milano : Adelphi, 1984, p. 246. 
1910, on estime ${ }^{9}$ que, sur ses 200000 habitants, la grande majorité (140000, soit $70 \%)$ est italienne, pour 25000 Slaves, principalement slovènes, et 9000 Autro-Allemands, qui forment une grande part de la très lourde administration impériale. Parmi ces communautés, il faut citer une importante communauté juive - phénomène banal en regard $\mathrm{du}$ reste de l'Empire, mais qui offre à Trieste un visage particulier ${ }^{10}$. Arrivés pour la plupart après 1719 - même si la présence d'un ghetto dans la vielle ville indique une présence plus ancienne, remontant au $\mathrm{XIV}^{\mathrm{e}}$ siècle -, les juifs triestins forment une population à la fois hétérogène - on y trouve des ashkénazes et des sépharades, des communautés très sécularisées et assimilées et d'autres plus religieuses, venues de Galicie ou de Corfou - et très homogène par les professions exercées ${ }^{11}$, mais surtout par son engagement politique et culturel en faveur de l'Italie $^{12}$ : une très grande majorité, en 1910, est italophone, tandis que peu utilisent l'allemand et un nombre infime le slovène. Cette communauté extrêmement bien intégrée est majoritairement libérale, laïque et irrédentiste. Le sionisme y est très peu représenté, et seules les familles les plus liées à la finance de Vienne ont un réel attachement dynastique. La communauté juive de Trieste semble un raccourci du destin de la ville et de son engagement dans la cause de l'Italie, pays dont elle ne sera, à son rattachement en 1918, qu'une périphérie. Car les Italiens de Trieste sont dans leur majorité violemment irrédentiste, c'est-à-dire partisans, par la voix du parti libéral-national, du rattachement au Royaume d'Italie. Or l'Autriche, après les lourdes pertes territoriales $\mathrm{du} \mathrm{XIX}^{\mathrm{e}}$ siècle, tient à conserver cette dernière portion de territoire italien, et l'un des Kronländer les plus anciens, qui forme son dernier débouché maritime.

C'est dans ce contexte tendu que paraît en 1909 dans la revue florentine La Voce, organe de l'avant-garde italienne, une série d'articles de Scipio Slataper intitulée Lettere triestine (Lettres triestines) ${ }^{13}$, et dont la première proclame que «Trieste n'a pas de tradition de culture » ( «Trieste non ha tradizioni di cultura »). Par ce titre provocateur et arrogant, Slataper, qui réside alors à Florence, s'en prend à l'autosatisfaction de la cité florissante qui, écrit-il, « se sent une ville importante » depuis qu'elle s'est « réveillée un jour entre une caisse d'agrumes et un sac de café ${ }^{14}$, et qui prétend devenir, à l'image des grandes cités com-

9. Michel David : La psicanalisi nella cultura italiana (note 4), p. 374.

10. Angelo Ara : «Juden und jüdisches Bürgertum im Triest der Jahrhundertwende », in: Andrei Corbea-Hoisie, Jacques Le Rider (Hrsg.): Metropole und Provinzen in Altösterreich. Wien, Köln, Weimar : Böhlau, 1996, p. 264-274.

11. La plupart sont marchands, avec un nombre croissant au XIX ${ }^{\mathrm{e}}$ siècle de professions libérales, médecins et avocats.

12. Ibid., p. 265-266.

13. Scipio Slataper : Lettere triestine, col seguito di altri scritti vociani di polemica su Trieste, postfazione di Elvio Guagnini, Trieste : Edizioni Dedolibri, 1988.

14. Ibid., p. 9 (je traduis). 
merçantes comme Venise ou Gènes, un nouveau centre de culture. Mais, selon Slataper, deux faits majeurs de l'histoire de Trieste ont empêché qu'elle vît se développer une tradition culturelle propre : l'esprit mercantile du port-franc, et l'afflux indifférencié de migrants dans une ville devenue un "refuge de malfaiteurs $»^{15}$. C'est l'existence de communautés séparées, sans lien entre elles et sans langue commune, sans rattachement ni attachement historique à la ville, qui différencie Trieste, à ses yeux, des centres de la culture italienne comme Florence. Le seul lien qui unit les habitants, le culte du dieu Mercure, est trop vil pour que la ville libre, si jalouse de ses privilèges douaniers, défende ses traditions, son dialecte et ses fêtes - italiennes :

C'est pourquoi l'histoire de Trieste est de glace, sans un seul élan d'idéalité, sans besoin d'art, sans inclination pour l'esprit. ${ }^{16}$

D'une telle ville sans tradition unifiée ni respect pour l'art et l'intellect, il ne peut y avoir qu'un exode qui contribue encore à la misère culturelle de la ville - et Slataper est lui-même un exemple paradigmatique de cet exil des meilleurs esprits qu'il déplore.

\section{L'axe Vienne - Florence, ou comment «se conquérir » (conquistarsi) une culture}

Si la Trieste italienne n'a effectivement pas la tradition de culture dont peuvent se targuer beaucoup de petites villes de la Péninsule, c'est bien que sa communauté italienne ne dispose pas des outils qui imposent, unifient, homogénéisent les forces culturelles d'une communauté : l'éducation et les relais culturels. Si l'Empire plurinational possède certes des écoles élémentaires dans les langues des sujets, de l'italien au ruthène, les lycées de Trieste sont des Staatsgymnasien austro-allemands dans lesquels la bourgeoisie envoie ses enfants afin qu'ils apprennent et se perfectionnent dans la langue administrative et dominante de l'Empire ${ }^{17}$. Le jeune Schmitz, futur Svevo, fait même ses études secondaires en Allemagne, dans un lycée de Stegnitz, près de Wurzbourg. Au moment précisément où la ville prétend se former une «tradition de culture », l'éducation secondaire n'est pas en mesure de donner aux jeunes Italiens de Trieste un enseignement littéraire de leur langue. Or,

15. Ibid., p. 10 (je traduis).

16. Ibid., p. 12: "Per ciò la storia di Trieste è ghiaccia, senza uno slancio di idealità, senza bisogna d'arte, senza affetto allo spirito. » Je traduis.

17. R. Bazlen : Scritti (note 8), p. 249: « Ti ho detto che frequentavo le scuole tedesche e che ero irredentista. La mia famiglia apparteneva a quella borghesia meno intellettuale della quale ti ho parlato. Dunque, scuole tedesche, come lo faceva la maggior parte della borghesia : era il periodo nel quale si sapeva che "chi sa le lingue ha il mondo in mano", come se ci fosse un bisogno illimitato di portieri d'albergo. » 
ce problème ne se règle pas non plus au niveau des études supérieures ${ }^{18}$. Après la perte de Pavie avec la Lombardie en 1859, puis de Padoue avec la Vénétie en 1866, l'Autriche doit renoncer aux deux universités italiennes de son territoire. Tandis que les Tchèques disposent, depuis 1882, de la partie tchèque de l'université caroline à Prague, les Polonais de celle de Cracovie, les Hongrois des établissements de Budapest et de Kolozsvár ${ }^{19}$ en Transylvanie et les Croates de l'université de Zagreb, la minorité italienne d'Istrie et de Dalmatie ne peut plus, après 1866, suivre d'études supérieures en italien sans sortir de l'Empire. En outre, cette sortie du territoire est rendue difficile par les tracasseries administratives, qui existaient du reste déjà pour les Istriens voulant s'inscrire à Padoue avant 1866, l'Autriche craignant que les universités italiennes ne soient des écoles de la révolte. Un tel exil ne peut être enfin qu'un acte de foi nationale, puisque les diplômes italiens ne sont pas reconnus dans la monarchie et que ces études en territoire italophone se révèlent donc inutiles, sinon suspectes, au retour en Istrie. C'est de cette situation que naît la italienische Universitätsfrage et l'une des revendications italiennes majeures au tournant du siècle : une université italienne à Trieste.

Des cours parallèles, notamment de droit, ont certes lieu en italien à l'université d'Innsbruck depuis 1864, et il a été question à plusieurs reprises de les détacher de cette université et de leur donner l'autonomie. Ce projet devient impossible à cause de la crainte des Tyroliens que leur territoire soit utraquisiert, comme le dit le joli terme administratif de l'époque, c'est-à-dire devienne officiellement bilingue ${ }^{20}$, comme la Bohême l'est devenue en 1897. De nombreux troubles sont organisés de 1901 à 1904 par les étudiants austro-allemands nationalistes et bloquent la situation qui ne trouve pas d'issue avant la Première Guerre mondiale. Les étudiants istriens n'ont donc d'autre choix que d'aller à Innsbruck, Graz et surtout Vienne pour recevoir une instruction académique, ce que fait la majorité de ceux qui souhaitent un diplôme scientifique, technique ou commercial - diplômes les plus « utiles » dans une ville vivant de son port. De ce fait, Trieste profite de la tradition humboldtienne des études à vocation scientifique et technique et se caractérise au début du XX $\mathrm{X}^{\mathrm{e}}$ siècle par un niveau d'instruction très élevé dans ces domaines, en comparaison de la situation dans le Royaume d'Italie.

Pour ceux qui se refusent à sacrifier ainsi aux exigences de la politique et du commerce maritime, l'alternative consiste à renoncer aux universités germanophones pour passer la frontière vers l'Italie, la plu-

18. Angelo Ara : «La questione dell'Università italiana in Austria », in : Ricerche sugli Austro-Italiani e l'Ultima Austria. Roma : Editrice Elia, 1974, p. 9-140.

19. Il s'agit de l'actuelle ville de Cluj-Napoca (en allemand : Klausenburg).

20. Le Tyrol de l'époque inclut en effet le Trentin italophone. 
part du temps pour Florence. C'est là le cas, exemplaire, de Carlo Michelstaedter, philosophe originaire de Gorizia (Görz) qui, en 1905, renonce à des études de mathématiques à Vienne pour se consacrer aux lettres à Florence. Comme lui, c'est donc tout un monde de jeunes Triestins, Slataper, les frères Stuparich, «Intellettuali di frontiera», comme les décrivait une exposition en 1983, qui, à l'image d'Alessandro Manzoni en 1827, viennent « rincer [leur] linge dans l'Arno » et s'engagent dans le cercle de La Voce pour un renouveau européen de la culture italienne. Svevo, qui n'a jamais pu faire ce pas, regretta toute sa vie de ne pas avoir pu «apprendre la langue aux sources vives ${ }^{21}$, en Toscane.

On a donc affaire, à Trieste, à une polarisation des choix professionnels, nationaux, linguistiques et politiques. C'est cette double culture, structurée par un axe Vienne - Florence, qui constitue la spécificité du milieu intellectuel de la ville. D'une part, la force du tropisme florentin s'explique par le problème linguistique des Triestins. Pour la plupart d'entre eux, le choix de l'italien comme langue d'écriture est en effet capital, mais également difficile : l'italien écrit est en quelque sorte une langue étrangère pour des dialectophones auxquels l'éducation secondaire et quelquefois les études supérieures n'ont pas permis d'apprendre de façon organique le standard littéraire. Cet effort linguistique de l'écrivain triestin s'exprime de façon aiguë chez Svevo, dont la formation est plus européenne qu'italienne, marquée par Shakespeare et Tourgueniev, Schopenhauer et le classicisme weimarien, au point qu'à son retour à Trieste, alors qu'il commence à travailler à la banque autrichienne Union, il fréquente assidûment la Biblioteca Civica car, écrit-il, «il s'agissait finalement de conquérir [conquistarsi] un peu de culture italienne ${ }^{22}$. Avec le recul, c'est le regret de ne pas être «pur»-purement italien, purement triestin même - qui se fait jour dans les écrits autobiographiques :

Serait-ce mon grand-père allemand qui m'empêche de mieux paraître latin? Et pourtant, j'ai toujours honoré et même étudié ma langue. Mais, depuis ma prime jeunesse,j'ai été ballotté dans les pays les plus divers, et en revanche, malgré un long désir, je n'ai connu Florence qu'à cinquante ans, et Rome à soixante. [...] Et c'est ainsi que la langue italienne pour moi est restée, définitivement, celle qui remue dans ma tête isolée. Les autres Triestins traînent avec eux leur dialecte, mais c'est un dialecte italien et ils sont plus purs que moi. ${ }^{23}$

21. Livia Veneziani-Svevo, citée par Elisabeth Schächter : Origins and identity : Essays on Svevo and Trieste, Leeds : Northern Universities Press, 2000, p. 17 : «Coltivava segretamente un sogno : studiare a Firenze per apprendere la lingua dalle vive fonti. »

22. I. Svevo : Profil autobiographique (note 1), p. 15. C'est principalement Carducci que Svevo découvre dans ces lectures tardives.

23. I. Svevo : lettre à Attilio Frescura du 10 janvier 1923, in : Écrits intimes (note 1), p. 216. 
Il est vrai que le purisme linguistique de la critique littéraire italienne a maintes fois reproché à Svevo «la pauvreté d'une langue farcie de solécismes et de formations dialectales ${ }^{24}$. L'écrivain reconnaît certes la nécessité de « refaire quelque peu une éducation littéraire si négligée ${ }^{25}$ afin que « le dialecte triestin s'affine ou disparaisse même complètement $»^{26}$, mais affirme en même temps le besoin d'écrire une 《langue vivante [qui] ne pouvait être que le dialecte triestin ${ }^{27}$. C'est une recherche de la «langue impossible » telle qu'il la décrit dans un article de 1889 : un toscan qui « a tout l'esprit du dialecte sans avoir [...] rien de bas ni de vulgaire $»^{28}$.

À l'autre bout de l'axe culturel qui traverse Trieste, c'est ce que Svevo nomme dans une lettre le «monde ouvert ${ }^{29}$ de Vienne, une culture européenne - ou mitteleuropéenne - ouverte sur d'autres aires linguistiques mais aussi sur d'autres domaines intellectuels. Car la même ville qui est linguistiquement reléguée à la périphérie de l'Italie se trouve paradoxalement située sur des axes de circulation intellectuelle intense. Son absence de tradition culturelle et sa situation périphérique donnent lieu à ce que Claudio Magris nomme la « presbytie intellectuelle » ${ }^{30}$ de Trieste, cette attention plus aiguë pour ce qui est loin que pour ce qui est proche, cette façon de regarder avec espoir les lointains tout en regrettant sa propre situation. C'est ainsi que la ville est l'avant-poste italien de la psychanalyse, notamment parce que les médecins triestins sont presque tous issus de la prestigieuse faculté de médecine de Vienne, à l'instar d'Edoardo Weiss, qui introduit la psychanalyse à Trieste aux alentours de $1910^{31}$. En outre, la communauté juive triestine s'avère être un relais extrêmement puissant de cette pénétration très rapide - Svevo découvre Freud au plus tard en 1911 - des théories freudiennes dans tous les cercles intellectuels de la ville ${ }^{32}$, dans lesquelles elles rencontrent certes des réserves, mais non les fortes réticences qu'elles provoquent, à la même époque et même plus tard, en Italie - on connaît les critiques de Benedetto Croce à l'encontre d'une science située «audelà des confins du monde et de la pensée qu'on nomme latins ${ }^{33}$. De

24. I. Svevo : Profil autobiographique (note 1), p. 14.

25. Ibid., p. 18.

26. Ibid., p. 22.

27. Ibid.

28. Italo Svevo : «Una commedia in lingua impossibile », in : L'Indipendente, Trieste, 2 avril 1884, in : Italo Svevo : Opera omnia, a cura di Bruno Maier, Milano : dall'Oglio editore, 1968 , vol. III p. 580.

29. I. Svevo : lettre à Ottavio Schmitz du 29 novembre 1895, in : Écrits intimes (note 1),

30. A. Ara et Cl. Magris : Trieste (note 3), p. 118.

31. Giorgio Voghera : «Gli anni della psicanalisi » [1968], in : Gli anni della psicanalisi, Pordenone : Edizioni Studio Tesi, 1980, p. 3-42.

32. M. David : La psicoanalisi nella cultura italiana (note 4), p. 375

33. Carlo Emilio Gadda : "Psicanalisi e letteratura », in : I Viaggi la morte, Milano 1958, p. 41. 
nombreux écrivains, tels Umberto Saba ${ }^{34}$ ou Roberto Bazlen ${ }^{35}$, sont en contact, voire en cure avec Edoardo Weiss.

Le milieu littéraire triestin se trouve très ouvert et attentif non seulement aux sciences, y compris aux sciences nouvelles, mais aussi à la pensée et à la littérature de l'Europe septentrionale, essentiellement de langue allemande: Trieste lit Nietzsche, Ibsen, Weininger, Hebbel, d'une façon qui est absente dans le reste de la Péninsule. Magris et Ara analysent ce phénomène paradoxal de «provincialisme d'avantgarde ${ }^{36}$ comme le résultat du déplacement de la grande culture allemande dans un univers linguistique et culturel complexe, vers

un espace géographiquement plus vaste au regard des cultures nationales et par conséquent spirituellement riche de différences, de tensions et de ferments qui lui donnent une ampleur d'horizons et de références tout à fait particulière dans la culture italienne de l'époque. ${ }^{37}$

\section{La caisse de résonance de la modernité : crise, réception, création}

La situation de la ville la rendait donc propre à être ce que Roberto Bazlen nomme une "caisse de résonance " ${ }^{38}$ de la conscience européenne. C'est là un des aspects les plus connus de la culture triestine, sismographe enregistrant et amplifiant la crise du début du siècle, même si cet aspect se détache sur le fond d' « une culture honnêtement épigonale, faite d'un mélange de traditions érudites et de passions nationales: [...] un humanisme provincial très comme il faut, honnête et vieillot. ${ }^{39}$ Trieste n'en reste pas moins, pour reprendre la définition de Bazlen dans une ébauche de 1934, une « ville de renonciations muettes et de tragédies inexprimées »:

L'homme, trop exposé à des influences diverses, [...] pôles opposés à l'extrême, d'où, à cause de la tension extrême, peut surgir l'étincelle la plus vive, étincelle qui ne trouve pas la forme et s'épuise dans le vain rongement du génie « impuissant », à qui la langue formée ne peut suffire et qui ne trouve pas la sienne propre :Däubler. ${ }^{40}$

34. Giorgio Voghera : Anni di Trieste. Gorizia : Editrice goriziana, 1989, p. 129. Saba sera même l'un des défenseurs de la psychanalyse contre Croce.

35. Renzo Cigoi : Quattrocento domande a un vecchio ebreo triestino. Colloqui con Giorgio Voghera, Roma : Semar Editore, 1996, p. 236-237.

36. M. David : La psicoanalisi nella cultura italiana (note 4), p. 379.

37. A. Ara et Cl. Magris : Trieste (note 3), p. 118.

38. R. Bazlen : Scritti (note 8), p. 252.

39. A. Ara et Cl. Magris : Trieste (note 3), p. 15.

40. R. Bazlen : Scritti (note 8), p. 237 : « L'uomo, troppo esposto a influenze diverse, [...] poli opposti al massimo, da cui, per la massima tensione, può sorgere la scintilla più viva, scintilla che non trova la forma e si esaurisce nel vano rosicchio del genio "impotente", cui la lingua formata non puo bastare e che non trova la propria : Däubler.» Je traduis. 
Cette caractérisation du poète expressionniste Theodor Däubler, luimême Triestin, s'applique à tous les grands écrivains de la ville, eux qui, au sens propre, ne maîtrisent pas les mots, mais dont les mots sont, selon l'expression de Svevo dans sa conférence consacrée à Joyce, « plutôt les maîtres que les esclaves ${ }^{41}$. Du reste, la recherche de la langue d'écriture, pour Svevo, dépasse le problème de l'identité nationale, abordé plus haut, pour devenir un véritable impératif moral, associé à la recherche de la sincérité et de la vérité, thèmes récurrents dans ses écrits intimes ${ }^{42}$ qui sont eux-mêmes des lieux où «faire l'exercice » de la sincérité. Écrivailler (scribacchiare) ${ }^{43}$ est pour lui la seule façon de ne pas mentir en écrivant, la seule façon de ne pas être victime de ces mots toscans dont Zeno dit, dans le roman de 1923, qu'avec chacun d'eux les Triestins mentent ${ }^{44}$. Des écrits intimes aux romans, c'est la même difficulté que Svevo met en exergue de son écriture - difficulté à être sincère, difficulté à trouver la forme stylistique adaptée à l'expression de cette vérité intime -, la même tension entre recherche morale et exigence stylistique. Si l'on considère la fréquence de ce genre de commentaires sous la plume de nombreux écrivains triestins, on peut affirmer avec Claudio Magris que la triestinité « exige une sincérité absolue des pages de sa littérature, parce que sans elles elle n'existerait pas ${ }^{45}$.

Ce qu'on a pu appeler sa moralità triestine ${ }^{46}$ - passion pour la vérité et méfiance envers le grand style qui caractérise également, à plus d'un titre, la modernité viennoise et pragoise -, naît d'une insécurité première liée à la langue, insécurité elle-même intimement liée à la situation politique de la ville. C'est ainsi que, par leur méfiance à l'encontre de la mise en forme proprement littéraire, les œuvres triestines apparaissent comme profondément tributaires de la modernité, alors même qu'elles sont en position marginale dans la culture italienne de l'époque et qu'on ne saurait leur attribuer une identité culturelle clairement définie. Or c'est dans ce même espace, et en lien profond avec ce sentiment d'insécurité face à la capacité de la langue à dire le vrai, que se déroule une autre expérience de la modernité : celle du dialogue d'égal à égal de la littérature avec les disciplines de la pensée, la philosophie et la science. Italo Svevo se présente à nouveau comme paradigme de cette expérience, avec Senilità (1902), inspiré, comme Les Buddenbrook de Thomas Mann, de Schopenhauer, et avec La Conscience de Zeno (1923), née, au moins en partie, d'un dialogue avec la psychanalyse freu-

41. I. Svevo : Écrits intimes (note 1), p. 301.

42. Ibid., p. 165 .

43. I. Svevo : Pages de Journal, 2 octobre 1899, in : Écrits intimes (note 1), p. 87.

44. I. Svevo : La Conscience de Zeno, trad. Paul-Henri Michel, éd. revue par Mario Fusco. Paris : Gallimard (coll. « Folio »), 1986, p. 497.

45. A. Ara et Cl. Magris : Trieste (note 3), p. 24.

46. Alberto Abruzzese : Svevo, Slataper e Michelstaedter. Lo stile e il viaggio, Venezia : Marsilio ed., 1979, p. 15. A. Abruzzese oppose la civiltà florentine, attentive au style et à la langue, à la moralità triestine, qui néglige la forme au profit du contenu. 
dienne. Or une telle écriture en dialogue est aussi un exercice de réception qui, à Trieste, a trouvé un terreau extrêmement fertile. Cette réception prend la forme d'une traduction double : il s'agissait en effet de franchir la barrière linguistique entre l'allemand, langue de Schopenhauer et de Freud, et l'italien, langue d'écriture, d'une part, et la frontière intellectuelle entre des disciplines extra-artistiques et la littérature, d'autre part. Dans ses écrits des années 1920, où il revient de façon réflexive sur sa pratique, Italo Svevo ne pense pas ce mouvement de traduction en termes d'opposition, mais bien en termes de conjonction, et même de «mariage »- union certes difficile parfois, mais féconde :

Ce rapport intime entre le philosophe et l'artiste, qui ressemble au mariage légal parce qu'ils ne s'entendent pas entre eux, exactement comme mari et femme, et cependant, comme mari et femme, font de très beaux enfants, apporte à l'artiste un renouvellement, ou, du moins, lui donne la chaleur et le sentiment d'une chose nouvelle, comme cela se produirait s'il était possible de changer une partie du vocabulaire et de nous donner des mots nouveaux, débarrassés des moisissures dues à leur vieillesse et au long usage qu'on en a fait. ${ }^{47}$

Par-delà les difficultés et par-delà la crise, par-delà la « situation délicate » de Trieste et des Triestins italophones, il y a donc la possibilité créatrice, la possibilité de franchir encore et encore les frontières qui traversent la ville même de Trieste et sa culture.

Il est permis de tirer du parcours et de l'œuvre singuliers d'Italo Svevo le paradigme d'un triple paradoxe de la culture triestine, prise dans les tensions de sa situation multiculturelle :

1. De l'absence de culture naît le constat d'une crise de la culture et une tentative très riche de dépasser celle-ci : le diagnostic de Slataper, qui faisait grief à la ville de sa composition par trop diverse, bigarrée et intellectuellement peu ambitieuse, s'avère pécher en ce qu'il ne prend pas en compte le fait que c'est justement dans une telle «culture de contact » (Michel David) que peuvent se résoudre les problèmes que pose le passage vers la modernité : absence de centralité, insuffisances des identités figées, éclatement des domaines de savoir.

2. Du problème central de la langue naît une littérature : «Trieste, plus peut-être que d'autres villes, est littérature, sa littérature; Svevo, Saba et Slataper sont moins des écrivains qui y sont nés, qui en sont nés, que des écrivains qui l'engendrent, qui la créent. ${ }^{48}$ Cependant ce n'est plus une littérature articulée autour de la création de la belle forme, mais une littérature alimentée par l'urgence de trouver un moyen d'être sincère, de retrouver la maîtrise des mots.

47. I. Svevo : Écrits intimes (note 1), p. 31.

48. A. Ara et Cl. Magris : Trieste (note 3), p. 23. 
3. C'est à la périphérie que se concentrent les questions centrales : c'est en effet dans une ville dont les habitants se sentent délaissés, à l'écart des flux de leurs cultures nationales respectives, que se trouvent réunies les conditions pour une vue de côté sur le monde qui est peutêtre un des traits saillants de la modernité centre-européenne. 